\title{
Optical bistability in axially modulated OmniGuide fibers
}

\author{
Marin Soljačić, Mihai Ibanescu, Steven G. Johnson, and J. D. Joannopoulos \\ Department of Physics, Massachusetts Institute of Technology, Cambridge, Massachusetts 02139
}

Yoel Fink

Department of Material Science and Engineering, Massachusetts Institute of Technology, Cambridge, Massachusetts 02139

Received June 4, 2002

\begin{abstract}
We demonstrate the feasibility of optical bistability in an axially modulated nonlinear OmniGuide fiber through analytical theory and detailed numerical experiments. At $1.55-\mu \mathrm{m}$ carrier wavelength, the in-fiber devices that we propose can operate with only a few tens of milliwatts of power, can have a nearly instantaneous response and recovery time, and can be shorter than $100 \mu \mathrm{m}$. (C) 2003 Optical Society of America

OCIS codes: $190.1450,230.4320$.
\end{abstract}

OmniGuide fibers are a new type of cylindrical multilayer dielectric fiber ${ }^{1,2}$ that have only very recently been implemented experimentally. ${ }^{3}$ Their cladding is an omnidirectional multilayer mirror that reflects light of any polarization and any direction. These photonic bandgap fibers can have a hollow core and a guiding mechanism that depends only on the cladding. We propose to exploit these facts to obtain much stronger axial optical modulation than is possible in conventional fibers through insertion of material (e.g., spheres) into the core. Moreover, because of strong transverse confinement, much smaller transverse modal areas are possible than in the usual low-index-contrast fibers. In this way, we show how optimal ultrafast bistable devices can be achieved with operating powers less than $50 \mathrm{~mW}$, whose highly nonlinear input-output power relation is key to many applications ${ }^{4}$ (e.g., all-optical pulse reshaping, optical limiting, logic gates). Our device retains all the advantages of similar photonic crystal ${ }^{5}$ or high-index-contrast devices ${ }^{6}$ in terms of power, size, and speed. However, the fact that it is an in-fiber device should make it easier to produce and to couple with another fiber. We solve the full nonlinear Maxwell equations numerically to demonstrate optical bistability in this class of system. Moreover, we present an analytical model that excellently describes the behavior of these systems and is very useful in predicting optimal designs.

A schematic of a typical design is shown in Fig. 1. It consists of an axially modulated single-mode OmniGuide fiber with a core of diameter $0.41 \lambda_{0}$, where $\lambda_{0}$ is the carrier wavelength in vacuum. The cladding consists of seven bilayers (periods), each $0.3 \lambda_{0}$ thick; $30 \%$ of the thickness is the high-index $\left(n_{H}=2.8\right)$ material. The innermost layer, adjacent to the core, is low index $\left(n_{L}=1.5\right)$. The axial modulation consists of 41 $\left(n_{\mathrm{SPH}}=1.5\right)$ spheres tightly filling the core (diameter $\left.0.41 \lambda_{0}\right)$. The periodicity of the axial modulation opens a bandgap for the propagating mode. Our threedimensional frequency-domain simulations ${ }^{7}$ tell us that structures such as the one in Fig. 1 easily open axial bandgaps of $6 \%$ or larger (versus $<0.1 \%$ in grated silica fibers).

A defect in the axial periodicity creates a resonant cavity supporting a tightly confined, high- $Q$ resonant mode. In the implementation of Fig. 1, the defect is introduced by a change of the index of refraction of the central sphere to $n_{\mathrm{DEF}}=1.9$. Tight confinement in the transverse direction is provided by the large bandgap of the OmniGuide cladding, while strong confinement in the axial direction is provided by the large axial bandgap. The cavity couples to the waveguide (axially uniform fiber) through tunneling processes. We model the low-index material to have an instantaneous Kerr nonlinearity [the index change is $\delta n(\mathbf{r}, t)=$ $c n_{L} \epsilon_{0} n_{2}|\mathbf{E}(\mathbf{r}, t)|^{2}$, where $n_{2}$ is the Kerr coefficient].

We perform nonlinear finite-difference time-domain simulations, ${ }^{8}$ with a perfectly matched layers boundary condition, of this system. These simulations propagate Maxwell equations exactly, with no approximations apart for the discretization. Because of the cylindrical symmetry of the system in Fig. 1, our system is effectively two dimensional. Consequently, we can obtain an excellent physical understanding of the system by performing two-dimensional finite-different time-domain simulations. The numerical values obtained with two-dimensional calculations will differ from the true three-dimensional values by only a geometrical factor of order unity.

The cavity supports a strongly localized resonant mode (transverse modal area $\approx \lambda_{0}{ }^{2} / 3$, axial length of mode $\approx 6 \lambda_{0}$ ). The system has a nearly Lorentzian transmission spectrum: $T(\omega) \equiv P_{\text {OUT }}(\omega) / P_{\mathrm{IN}}(\omega) \approx$ $\gamma_{W}^{2} /\left[\left(\gamma_{R}+\gamma_{W}\right)^{2}+\left(\omega-\omega_{\mathrm{RES}}\right)^{2}\right]$, where $P_{\text {OUT }}$ and $P_{\text {IN }}$ are the outgoing and incoming power, respectively; $\omega_{\mathrm{RES}}$ is the resonant frequency; $\gamma_{W}$ is the decay width that is due to the coupling of the cavity mode to the waveguides; and $\gamma_{R}$ is the decay width that is due to the coupling to the cladding modes. ${ }^{9}$ We measure a quality factor $Q=\omega_{\mathrm{RES}} /\left[2\left(\gamma_{R}+\gamma_{W}\right)\right]=540$ and a peak transmission $T_{P}=0.88$; from this we
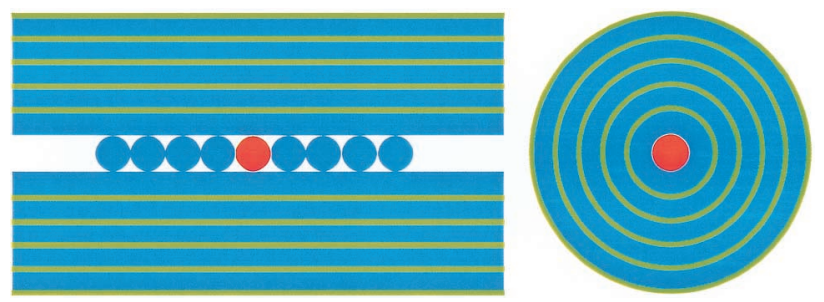

Fig. 1. Schematic of a nonlinear OmniGuide device in which we demonstrate optical bistability. Left, longitudinal cross section; right, transverse cross section. 
obtain the result that the radiation quality factor $Q_{R}=\omega_{\mathrm{RES}} / 2 \gamma_{R}=8700 . \quad Q_{R}$ is finite because of the coupling of energy to the radiating cladding modes; this coupling is the primary cause of losses in our system but can be controlled. ${ }^{10}$

First, we perform numerical experiments in which we launch a series of Gaussian pulses into the system. We choose a carrier frequency $\omega_{0}=\omega_{\mathrm{RES}}-$ $3.2\left(\gamma_{W}+\gamma_{R}\right)$, and the FWHM bandwidth of our pulses is $\omega_{0} / \mathrm{FWHM}=796$. The ratio of the transmitted $\left(E_{\text {OUT }}\right)$ versus the incoming $\left(E_{\text {IN }}\right)$ pulse energy increases sharply as we approach the bistability threshold and decreases after the threshold is passed, as shown in the top left-hand panel of Fig. 2. Transmitted pulse spectra are also shown for a few pulses in the top right-hand panel of Fig. 2; the nonlinear cavity redistributes the energy in the frequency spectrum. If these changes to the spectrum are undesirable, one can remove them by optimizing the device, using timeintegrating nonlinearity, or by adding a frequencydependent filter to the output of the device. Typical output-pulse shapes are shown in the lower panels of Fig. 2. As one can see from the bottom left-hand panel, even without optimizing our system we still obtain well-behaved shapes of output pulses in the regime where $E_{\text {OUT }} / E_{\text {IN }}$ is maximal.

For the case of cw signals, we can achieve a precise analytical understanding of bistability in this system, making use of a new fundamental dimensionless characteristic of the cavity, nonlinear feedback parameter $\kappa$ (derived in detail in Ref. 5):

$\kappa \equiv\left(\frac{c}{\omega_{\mathrm{RES}}}\right)^{d}$

$\times \frac{\int_{\mathrm{VOL}} \mathrm{d}^{d} \mathbf{r}\left[|\mathbf{E}(\mathbf{r}) \cdot \mathbf{E}(\mathbf{r})|^{2}+2\left|\mathbf{E}(\mathbf{r}) \cdot \mathbf{E}^{*}(\mathbf{r})\right|^{2}\right] n^{2}(\mathbf{r}) n_{2}(\mathbf{r})}{\left.\left[\int_{\mathrm{VOL}} \mathrm{d}^{d} \mathbf{r}|\mathbf{E}(\mathbf{r})|^{2} n^{2}(\mathbf{r})\right]^{2} n_{2}(\mathbf{r})\right|_{\mathrm{MAX}}}$,

where $n(\mathbf{r})$ is the unperturbed index of refraction, $\mathbf{E}(\mathbf{r}, t)=\left[\mathbf{E}(\mathbf{r}) \exp (i \omega t)+\mathbf{E}^{*}(\mathbf{r}) \exp (-i \omega t)\right] / 2$ is the electric field, $n_{2}(\mathbf{r})$ is the local Kerr coefficient, volume (VOL) of integration is over the extent of the mode, and $d$ is the dimensionality of the system in question. As can be seen from Eq. (1), $\kappa$ is dimensionless and scale invariant. It is determined by the degree of spatial confinement of the field in the nonlinear material. To an excellent approximation, $\kappa$ is independent of $n_{2}$, the peak electric field amplitude, $Q$, and small deviations in $\omega_{0}$. Let us denote as $P_{\text {IN }}{ }^{S}$ and $P_{\text {OUT }} S$ the steady-state values of $P_{\mathrm{IN}}$ and $P_{\mathrm{OUT}}$, respectively. Using a Lorentzian transmission spectrum in the linear case and perturbation theory for small $\delta n(\mathbf{r})$, we obtain

$$
\frac{P_{\mathrm{OUT}} S}{P_{\mathrm{IN}} S}=\frac{T_{P}}{1+\left[\left(P_{\mathrm{OUT}} S / P_{0}\right)-\delta\right]^{2}},
$$

where $\delta=\left(\omega_{\mathrm{RES}}-\omega_{0}\right) /\left(\gamma_{W}+\gamma_{R}\right), T_{P}=\left[\gamma_{W}{ }^{2} /\left(\gamma_{W}+\right.\right.$ $\left.\left.\gamma_{R}\right)^{2}\right]$ is the peak transmission, and $P_{0}$ is a characteristic power of this cavity given by

$$
P_{0} \equiv \frac{\sqrt{T_{P}}}{\left.\kappa Q^{2}\left(\omega_{\mathrm{RES}} / c\right)^{d-1} n_{2}(\mathbf{r})\right|_{\mathrm{MAX}}} .
$$

According to Eq. (2), $P_{0}$ sets the power scale for observing bistability in a cavity of interest.

To check our analytical theory we obtain $\kappa=0.010$ from a single nonlinear computation; together with the knowledge of $Q$ and $\omega_{\text {RES }}$, we obtain $P_{\mathrm{OUT}}{ }^{S}\left(P_{\mathrm{IN}}{ }^{S}\right)$ for $\delta=3.2$, which we plot as a solid curve in Fig. 3. We compare our analytical theory with numerical experiments in which we launch smoothly turned on cw signals into the cavity. To observe the upper hysteresis branch we launch large-amplitude and wide Gaussian pulses that decay into smaller steady-state $\mathrm{cw}$ values. The small discrepancy between our analytical theory and the numerical experiments in Fig. 3 is attributable mostly to the fact that the linear-regime transmission curve is not a perfect Lorentzian.

Although numerical simulations for larger $Q$ would be prohibitively long, our analytical model allows us to predict the behavior of a high- $Q$ device. According
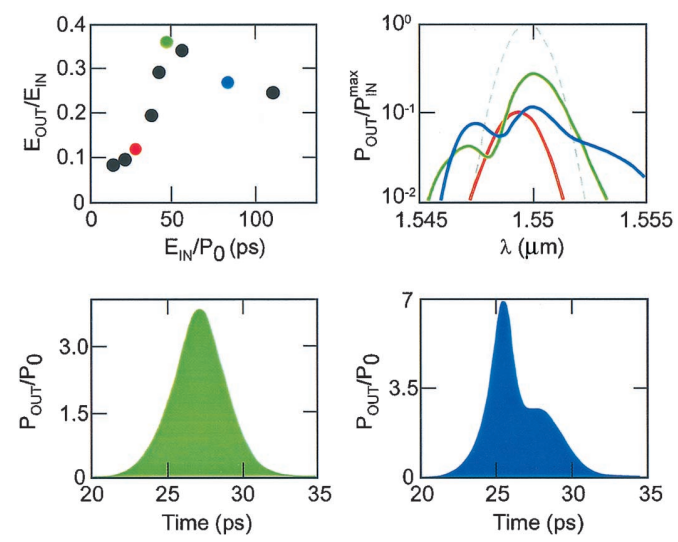

Fig. 2. Numerical experiments with launching temporal Gaussian pulses into the nonlinear system of Fig. 1. The top left-hand panel shows the transmission as a function of the incoming pulse energy. The colored curves in the top right-hand panel display the output spectra for a few pulses, corresponding to the colored dots in the top left-hand panel. The spectrum of each pulse is normalized to its peak incoming power; i.e., the input pulse, normalized in the same manner, is displayed with the gray dashed curve. The bottom panels show the output shapes for the incoming pulses, denoted by the green and blue dots, respectively, in the top left-hand panel.

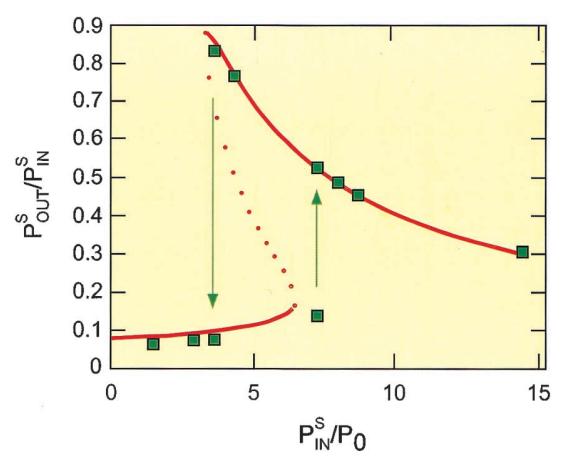

Fig. 3. Plot of the observed $P_{\mathrm{OUT}}{ }^{S}$ versus $P_{\mathrm{IN}}{ }^{S}$ for the device from Fig. 1, when $\delta=3.2$. The green squares are points obtained from numerical experiments. The red curve is the analytical prediction, which clearly matches the numerical experiments; the dotted part of the curve is unstable and therefore physically unobservable. 
to Eq. (3), the power requirements drop with $1 / Q^{2}$. $Q$ can be increased by addition of more spheres to the walls of the cavity. For $Q$ of 6000 (which is still compatible with 10-Gbit/s signals), the nonlinear index changes are $<0.001$ (which is still compatible with nearly instantaneous nonlinear materials). The power needed to observe bistability is $50 \mathrm{~mW}$ (assuming $n_{2}=2 \times 10^{-17} \mathrm{~m}^{2} / \mathrm{W}$, a value typical for the materials that we are using), which is fairly close to the 5-mW single-channel peak power levels used in telecommunications. The power can be further decreased by reduction of the modal volume and (or) by use of materials with a larger Kerr coefficient.

There are two reasons why the required power is so small compared with that of other fiber systems that display optical bistability. ${ }^{11,12}$ First, all the energy of the mode is concentrated in a very small modal volume $\left(\approx 2 \lambda^{3}\right)$; consequently, for the same amount of modal energy, the peak $\delta n(\mathbf{r})$ induced in the system is much larger than in other systems with much larger modal volumes. ${ }^{12}$ This is manifested by the fact that the nonlinear feedback parameter $\kappa=0.010$ is fairly large. [For comparison, if one had a system in which all the energy of the mode were contained uniformly inside a volume $\left(\lambda_{0} / 2 n_{L}\right)^{3}, \kappa$ would be $\approx 0.15$.] Second, a large quality factor can be achieved at the same time as the large $\kappa$. Increasing $Q$ while keeping other parameters fixed decreases the power requirements as $1 / Q^{2}{ }^{13,14}$ The first factor of $Q$ appears because increasing $Q$ for a fixed $P_{\text {IN }}$ leads to a larger peak electric field inside the cavity, because of the longer energy accumulation. The second factor of $Q$ comes from the fact that the resonance peak width is $\sim 1 / Q$, thereby reducing the required frequency (index) shift by $1 / Q$ as well.

On the experimental front, so far we have been successful in inserting $d=1.75 \mu \mathrm{m}$ spheres into $d=$ $2 \mu \mathrm{m}$ core fiber via capillary action (optical tweezers could be used instead). ${ }^{15}$ To our advantage, spheres tend to stick together because of van der Waals forces. As a next step, we plan to decrease the pressure inside the core and heat the fiber to the melting point so that it will clasp the spheres tightly; this is a standard technique usually used to decrease the core radii of hollow-core fibers. Clearly, there are many other possibilities for implementing axial modulation. Conceptually, the fact that the guiding mechanism does not depend on the core and the fact that the core is initially hollow provide for significantly more experimental flexibility in implementing a stronger optical axial modulation than exists in conventional fibers. Other authors ${ }^{16}$ demonstrated impressive manipulation of optical properties of holey fibers, including periodic axial modulation of the core, by inserting various kinds of materials into the holes. Alternatively, one could perform photolitography on the inner surface of the core: A photoresist would be codrawn as the innermost layer of the cladding, one would shine laser beams from the side to implement cross linking of the photoresist, and then one would use the hollow core to transport all the acids and (or) bases needed to etch a periodic structure onto the innermost layer of the cladding.
We emphasize that, since the transverse confinement principle is not index guiding, periodic axial modulation is not the only way to achieve axial confinement in OmniGuide fibers. In fact, simply shrinking the core radius ${ }^{16}$ (or equivalently decreasing the index of the core) of an OmniGuide fiber can shift the cutoff frequency of the operating mode above the operating frequency. Similarly to what occurs in a metallic waveguide, a mode experiences exponential decay once it encounters such a region; this property is sufficient for implementing all the ideas presented above. Finally, one should be able to implement all the principles described in this Letter in any hollow photonic crystal fiber that has a large enough lateral bandgap.

Help from S. Jacobs, T. Engeness, O. Weisberg, and M. Skorobogatiy of OmniGuide Communications, Inc., and from E. Ippen of the Massachusetts Institute of Technology is gratefully acknowledged. This work was supported in part by the Materials Research Science \& Engineering Center program of the National Science Foundation under grant DMR-9400334. M. Soljačić's e-mail address is marin@alum.mit.edu.

\section{References}

1. Y. Fink, D. J. Ripin, S. Fan, C. Chen, J. D. Joannopoulos, and E. L. Thomas, J. Lightwave Technol. 17, 2039 (1999).

2. S. G. Johnson, M. Ibanescu, M. Skorobogatiy, O. Weisberg, T. D. Engeness, M. Soljačić, S. A. Jacobs, J. D. Joannopoulos, and Y. Fink, Opt. Express 9, 748 (2001), http://www.opticesexpress.org.

3. S. D. Hart, G. R. Maskaly, B. Temelkuran, P. H. Prideaux, J. D. Joannopoulos, and Y. Fink, Science 296, 511 (2002).

4. B. E. A. Saleh and M. C. Teich, Fundamentals Of Photonics (Wiley, New York, 1991).

5. M. Soljačić, M. Ibanescu, S. G. Johnson, Y. Fink, and J. D. Joannopoulos, Phys. Rev. E 66, 055601(R) (2002).

6. J. S. Foresi, P. R. Villeneuve, J. Ferrera, E. R. Thoen, G. Steinmeyer, S. Fan, J. D. Joannopoulos, L. C. Kimerling, H. I. Smith, and E. P. Ippen, Nature 390, 143 (1997).

7. S. G. Johnson and J. D. Joannopoulos, Opt. Express 8, 173 (2001), http://www.opticesexpress.org.

8. For a review, see A. Taflove, Computational Electrodynamics: The Finite-Difference Time-Domain Method (Artech House, Norwood, Mass., 1995).

9. See, e.g., S. Fan, P. R. Villeneuve, J. D. Joannopoulos, and H. A. Haus, Phys. Rev. B 64, 245302 (2001), and H. A. Haus, Waves And Fields in Optoelectronics (Prentice-Hall, Englewood Cliffs, N.J., 1984).

10. S. G. Johnson, S. Fan, A. Mekis, and J. D. Joannopoulos, Appl. Phys. Lett. 78, 3388 (2001).

11. S. Coen and M. Haelterman, Opt. Lett. 24, 80 (1999).

12. S. Radić, N. George, and G. P. Agrawal, J. Opt. Soc. Am. B 12, 671 (1995).

13. A. Yariv, IEEE Photon. Technol. Lett. 14, 483 (2002).

14. J. E. Heebner and R. Boyd, Opt. Lett. 24, 847 (1999).

15. B. Temelkuran, Massachusetts Institute of Technology, Cambridge, Mass. 02139. (personal communication, November, 2001).

16. C. Kerbage and B. J. Eggleton, Opt. Photon. News 13(9), 38 (2002). 